\title{
On the physical DGLAP evolution of structure functions and its use for detecting saturation effects*
}

\author{
Martin Hentschinski ${ }^{\dagger}$ \\ Instituto de Ciencias Nucleares \\ Universidad Nacional Autónoma de México \\ Apartado Postal 70-543 \\ México D.F. 04510 MX \\ E-mail: hentschinski@correo.nucleares.unam.mx
}

Physical anomalous dimensions are a formulation of the DGLAP evolution of Deep Inelastic structure functions which is indepedent of factorization scheme and -scale. In this proceedings we provide an outlook on possible applications, in particular in the search of saturation effects. As an original contribution we present a short study of the scale dependence of physical evolved structure functions for large initial scale $Q_{0}^{2}=30 \mathrm{GeV}^{2}$

XXIII International Workshop on Deep-Inelastic Scattering,

27 April - May 12015

Dallas, Texas

${ }^{*}$ Work in collaboration with Marco Stratmann

† Speaker. 


\section{Introduction}

Analysis of Deep-inelastic scattering (DIS) cross sections is generally performed through global fits of scale-dependent quark and gluon distribution functions $f_{i}\left(x, Q^{2}\right), i=q, \bar{q}, g$. The underlying theoretical framework of such analysis is based on the collinear factorization theorem [1], which organizes the computation of DIS structure functions $F_{2, L}\left(x, Q^{2}\right)$ into the convolution of short-distance Wilson coefficients and long-distance parton distribution functions (PDFs) (see e.g. [2] for a recent review). In order to formulate such a theorem it is necessary to introduce a factorization scale $\mu_{f}$ which separates long- and short-distance physics. Independence of physical observables on $\mu_{f}$ allows then to derive renormalization group equations (RGEs) which govern the scale dependence of PDFs, known as the Dokshitzer-Gribov-Lipatov-Altarelli-Parisi (DGLAP) equations. Since there is an infinite number of different ways to realize factorization, one is left with an additional choice of the factorization scheme with the $\overline{\mathrm{MS}}$ prescription the generally adapted one. For observables, such as DIS structure functions, any residual dependence on factorization scheme and - scale $\mu_{f}$ is suppressed by an additional power of $\alpha_{s}$, i.e., is formally one order higher in the perturbative expansion but not necessarily numerically small.

As an alternative to this conventional treatment it is also possible to formulate QCD scale evolution equations directly for observables without referring to auxiliary, convention-dependent PDFs, which avoids introduction of an artificial factorization scheme and -scale dependence altogether [3]. The framework is suited best for theoretical analyses of DIS data; in particular one remains in this case with the renormalization scale $\mu_{r}$ as the only theoretical ambiguity. Since theory uncertainties are in this way reduced to a minimum, it is therefore this 'physical' formulation of DGLAP evolution which is most suitable for extractions of $\alpha_{s}$ from inclusive DIS data (for a first study see e.g. [4]). Moreover, physical evolution allows for the most stringent tests of DGLAP evolution itself. This is of particular interest for regions of phase space where a breakdown of collinear factorization is expected, such as the limit $x \rightarrow 0$ of DIS structure functions, where large parton densities eventually saturate and require a more complete description including terms usually suppressed by powers of $Q^{2}$. A potential application of physical evolution for such studies is illustrated and discussed in Fig. 1. The outline of these proceedings is as follows: in Sec. 2 we provide some details on the derivation and definition of physical evolution kernels while Sec. 3 presents an analysis of the remaining renormalization scale dependence of physical evolution up to next-to-next-to-leading order (NNLO) in $\alpha_{s}$ for large input scales $Q^{2}=30 \mathrm{GeV}^{2}$. For more details we refer the interested reader to [5].

\section{Physical evolution kernel}

To define physical evolution kernels we use that the $x$-space convolutions of coefficient functions and PDFs turn into products in conjugate Mellin space, $a(n)=\int_{0}^{1} d x x^{n-1} a(x)$. Moments of DIS structure functions $F_{I}\left(x, Q^{2}\right)$ can then be expressed as

$$
F_{I}\left(n, Q^{2}\right)=\sum_{k} C_{I, k}\left(n, \alpha_{s}\left(\mu^{2}\right), \frac{Q^{2}}{\mu^{2}}, \frac{\mu_{r}^{2}}{\mu_{f}^{2}}\right) \cdot f_{k}\left(n, \alpha_{s}\left(\mu^{2}\right), \frac{\mu_{f}^{2}}{Q_{0}^{2}}, \frac{\mu_{r}^{2}}{\mu_{f}^{2}}\right) .
$$



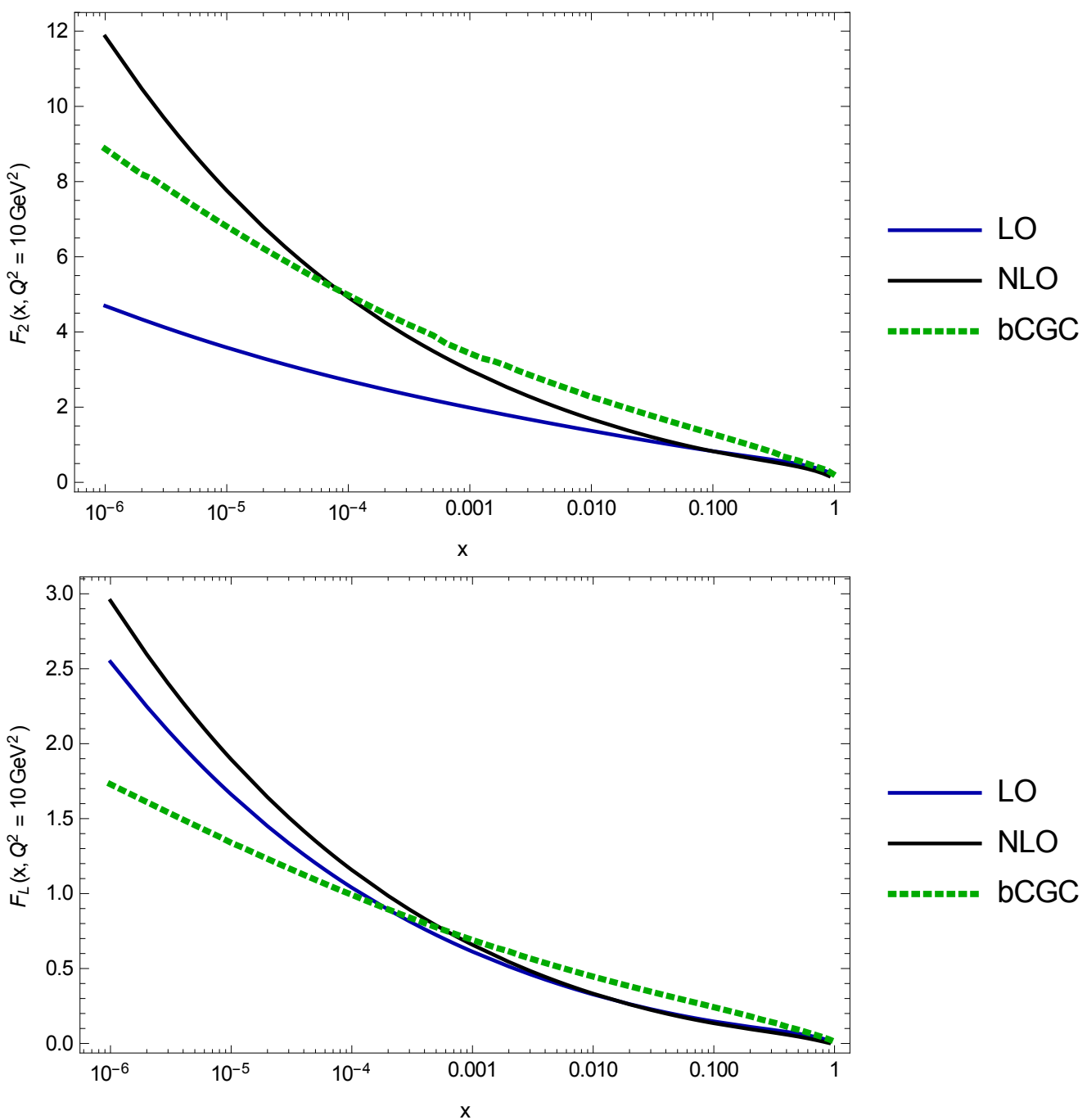

LO

NLO

bCGC

Figure 1: Gold structure functions $F_{2}$ and $F_{L}$ have been calculated at $Q^{2}=2 G e V^{2}$ from the bCGC-model, using the fit [6] to HERA proton DIS data, combined with a scaling $Q_{S}^{2} \rightarrow Q_{S}^{2} A^{1 / 3}$. The result has been fitted and used as input for physical DGLAP evolution. The plots show a comparison of physical DGLAP evolution at leading $(L O)$ and next-to-leading (NLO) order for the doublet $\left(F_{2}, F_{L}\right)$ from $Q_{0}^{2}=2 \mathrm{GeV}^{2} \rightarrow Q^{2}=10 \mathrm{GeV}$ and the corresponding $b C G C$ result at $Q^{2}=10 G e V^{2}$.

The sum runs over all contributing quark flavors and the gluon, each represented by a $\operatorname{PDF} f_{k}$. The non-perturbative PDFs $f_{k}\left(n, \mu^{2}\right)$ obey the DGLAP evolution equations

$$
\frac{d f_{k}\left(n, \mu^{2}\right)}{d \ln \mu^{2}}=\sum_{l} P_{k l}\left(n, \alpha_{s}\left(\mu^{2}\right), \frac{Q^{2}}{\mu^{2}}\right) f_{l}\left(n, \mu^{2}\right),
$$

while coefficient functions $C_{I, k}[7,8,9]$ and splitting kernels $P_{k l}[10,11,12]$ can be calculated in perturbative QCD and exhibit the following expansion in $\alpha_{s}$

$$
P_{k l}=\sum_{m=0}\left(\frac{\alpha_{s}}{4 \pi}\right)^{1+m} P_{k l}^{(m)}(n), \quad C_{I, k}=\sum_{m=0}\left(\frac{\alpha_{s}}{4 \pi}\right)^{m_{0}+m} C_{I, k}^{(m)}(n),
$$


where $m_{0}$ depends on the first non-vanishing order in $\alpha_{s}$ in the expansion for the observable under consideration, e.g. $m_{0}=0$ for $F_{2}$ and $m_{0}=1$ for $F_{L}$. The DGLAP evolution equation are formulated as $n_{f}-1$ evolution equations for the different non-singlet quark flavor combinations and a $2 \times 2$ matrix valued evolution equation, which evolves the flavor singlet vector $(\Sigma, g) ; g\left(n, \mu^{2}\right)$ the gluon distribution and $\Sigma\left(n, \mu^{2}\right)=\sum_{f}^{n_{f}}\left[q_{f}\left(n, \mu^{2}\right)+\bar{q}_{f}\left(n, \mu^{2}\right)\right]$ the quark flavor singlet. In the following we concentrate ourselves on the flavor singlet sector only; for the physical evolution of the nonsinglet sector see e.g. [4]. Using that any doublet of flavor singlet observables $F=\left(F_{A}, F_{B}\right)$ is related to the flavor singlet vector $(\Sigma, g)$ through a coefficient matrix $C$

$$
C=\left(\begin{array}{cc}
C_{A q} & C_{A g} \\
C_{B q} & C_{B g}
\end{array}\right) \quad P=\left(\begin{array}{cc}
P_{q q} & P_{q g} \\
P_{g q} & P_{g g}
\end{array}\right),
$$

and introducing further a corresponding $2 \times 2$ matrix $P$ for the matrix-valued kernel of the DGLAP evolution in the flavor singlet sector, one finds in a straight forward manner

$$
\frac{d F\left(n, Q^{2}\right)}{d \ln Q^{2}}=\left(4 \pi \beta \frac{d C}{d \alpha_{s}} C^{-1}+C \cdot P \cdot C^{-1}\right) \cdot F
$$

where we made in addition use of the RG equation of $\alpha_{s}$ governed by the QCD beta function

$$
\frac{d \alpha_{s}(\mu)}{d \ln \mu^{2}}=4 \pi \beta\left(a_{s}\right)=-\alpha_{s} \sum_{m}\left(\frac{\alpha_{s}}{4 \pi}\right)^{m+1} \beta_{m} .
$$

The resulting physical evolution kernels

$$
K\left(\alpha_{s}\left(\mu_{r}^{2}\right), \frac{Q^{2}}{\mu_{r}^{2}}\right) \equiv\left(\beta \frac{d C}{d a_{s}} C^{-1}+C \cdot P \cdot C^{-1}\right)=\frac{\alpha_{s}\left(\mu_{r}^{2}\right)}{4 \pi} \sum_{m=0}\left(\frac{\alpha_{s}\left(\mu_{r}^{2}\right)}{4 \pi}\right)^{m} K^{(m)}\left(n, \frac{Q^{2}}{\mu_{r}^{2}}\right),
$$

are independent of factorization scheme and -scale [3] with the renormalization scale $\mu_{r}$ as their only remaining scale ambiguity at finite perturbative order.

\section{Renormalization scale dependence}

To determine the renormalization scale dependence of physical evolution kernels we use kernels calculated at $\mu_{r}=Q$, see e.g. [5], and recover their full renormalization scale dependence using the same prescription as for conventional DGLAP splitting kernels i.e. by Taylor expanding $\alpha_{s}\left(Q^{2}\right)$ in terms of $\alpha_{s}\left(\mu_{r}^{2}\right)$; see e.g. [13] for a discussion in the case of splitting kernels. For the following numerical study we fix $n_{f}=4$ and use a realistic toy input at $Q^{2}=30 \mathrm{GeV}^{2}$ for quark singlet and gluon distribution [14],

$$
\begin{aligned}
& x \Sigma(x)=0.6 x^{-0.3}(1-x)^{3.5}\left(1+5 x^{0.8}\right) \\
& x g(x)=1.6 x^{-0.3}(1-x)^{4.5}\left(1-0.6 x^{0.3}\right),
\end{aligned}
$$

from which we calculate structure functions using LO coefficients, independent of the actual studied perturbative order. The strong coupling is fixed to $\alpha_{s}\left(Q_{0}\right)=0.2$. We study as examples the flavor singlet sector of the doublets $\left(F_{2}, F_{L}\right)$ and $\left(F_{2}, F_{S}\right)$ with $F_{S}$ the $F_{2}$ scaling violations

$$
F_{S}\left(x, Q^{2}\right) \equiv \frac{d F_{2}\left(x, Q^{2}\right)}{\ln Q^{2}}
$$



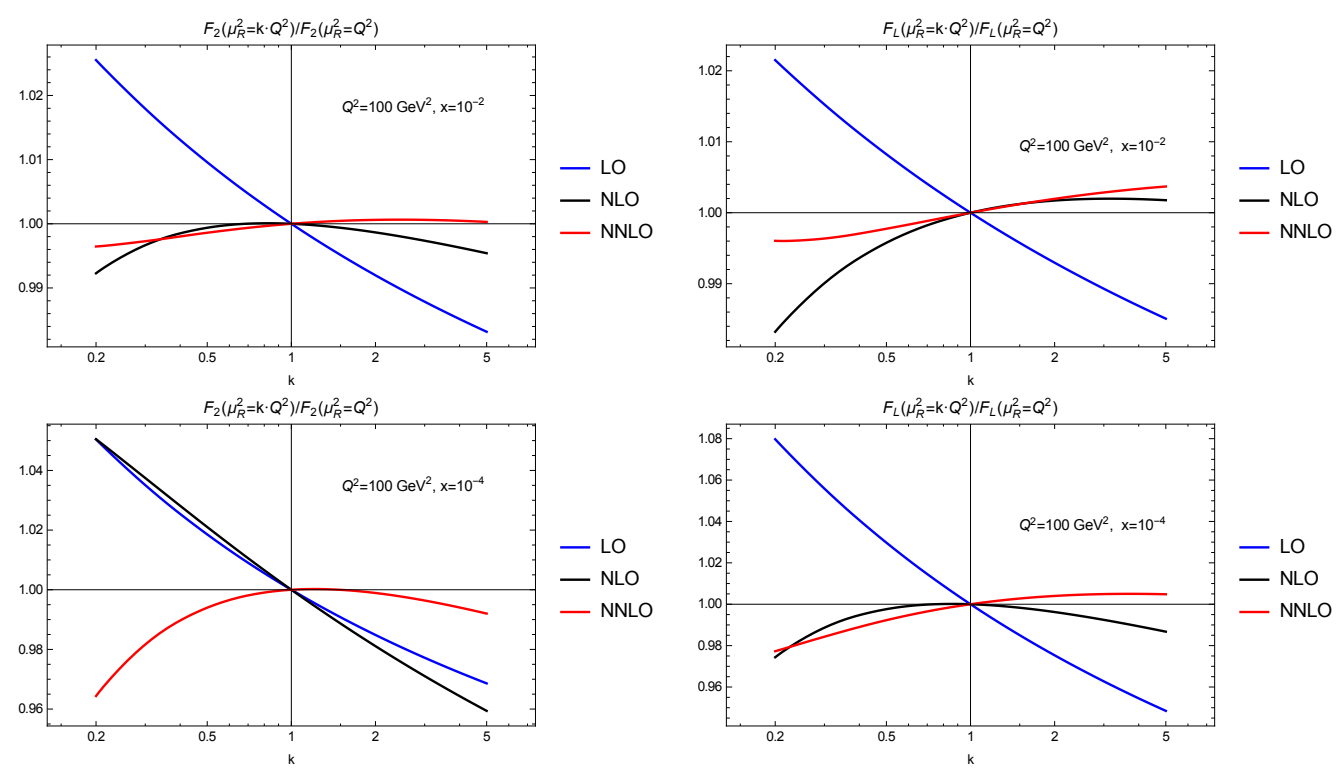

Figure 2: Renormalization scale dependence of the doublet $\left(F_{2}, F_{L}\right)$
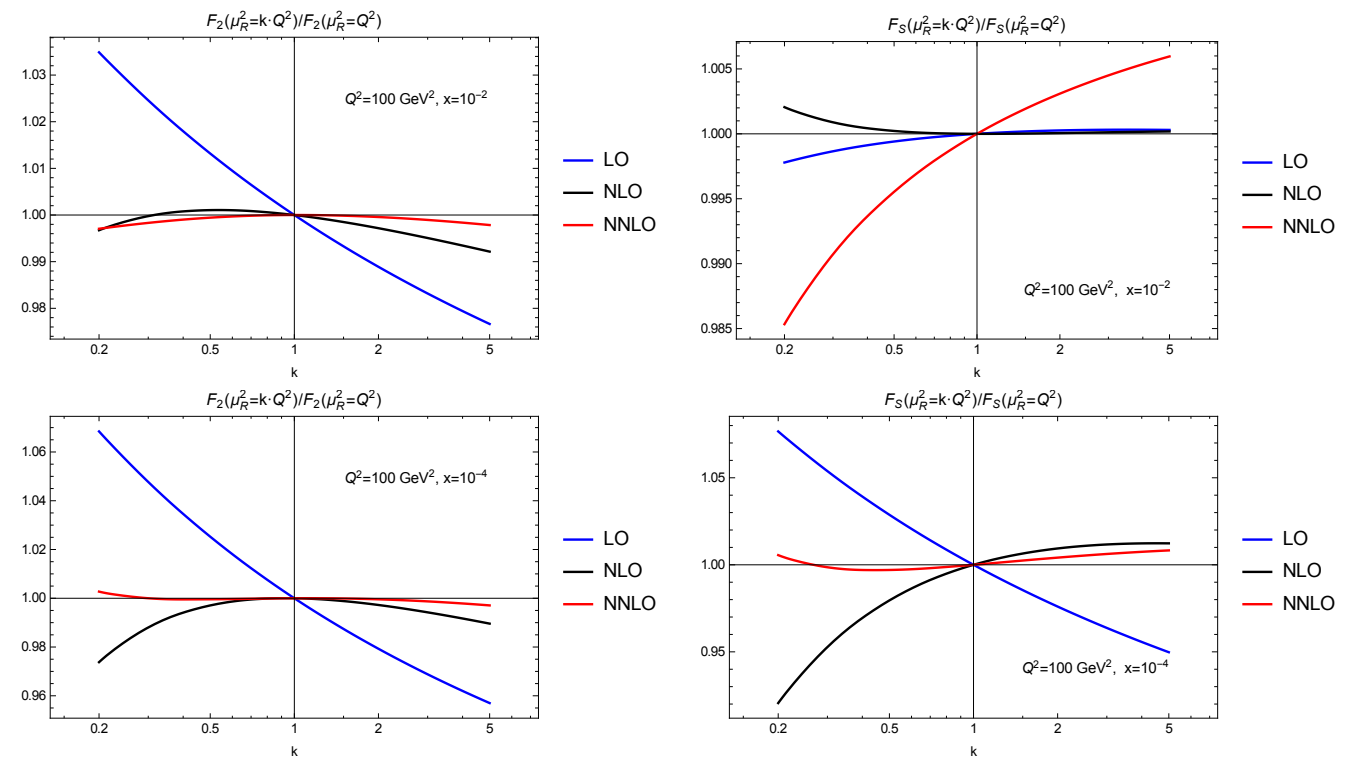

Figure 3: Renormalization scale dependence of the doublet $\left(F_{2}, F_{S}\right)$

Our result are depicted in Fig. 2 and Fig. 3 and reveal a very small dependence on the chosen renormalization at NNLO, with the doublet $\left(F_{2}, F_{S}\right)$ slightly less sensitive to the variation of the renormalization scale. Note that the variations have been performed over a very wide range i.e. $\mu_{r, 0}^{2} / \mathrm{GeV}^{2} \in[6,150]$ and $\mu_{r}^{2} / \mathrm{GeV}^{2} \in[20,500]$ respectively.

In conclusion we find a very mild dependence on the renormalization scale, if the initial scale for DGLAP evolution is rather large. For the case of small initial scales we refer to the discussion in [5]. 


\section{Acknowledgments}

This work was supported in part by UNAM-DGAPA-PAPIIT grant number 101515 and CONACyTMexico grant number 128534.

\section{References}

[1] J. C. Collins, D. E. Soper and G. F. Sterman, Adv. Ser. Direct. High Energy Phys. 5, 1 (1989) [hep-ph/0409313].

[2] J. Blumlein, Prog. Part. Nucl. Phys. 69, 28 (2013) [arXiv:1208.6087 [hep-ph]].

[3] W. Furmanski and R. Petronzio, Z. Phys. C 11, 293 (1982), S. Catani, Z. Phys. C 75, 665 (1997), J. Blumlein, V. Ravindran, and W. L. van Neerven, Nucl. Phys. B 586, 349 (2000).

[4] W. L. van Neerven and A. Vogt, Nucl. Phys. B 568, 263 (2000) [hep-ph/9907472].

[5] M. Hentschinski and M. Stratmann, arXiv:1311.2825 [hep-ph].

[6] A. H. Rezaeian and I. Schmidt, Phys. Rev. D 88 (2013) 074016 [arXiv:1307.0825 [hep-ph]].

[7] W. L. van Neerven and E. B. Zijlstra, Phys. Lett. B 272, 127 (1991); Phys. Lett. B 273, 476 (1991); Nucl. Phys. B 383, 525 (1992).

[8] S. Moch and J. A. M. Vermaseren, Nucl. Phys. B 573, 853 (2000).

[9] D. I. Kazakov and A. V. Kotikov, Nucl. Phys. B 307, 721 (1988) [Erratum-ibid. B 345, 299 (1990)]; D. I. Kazakov, A. V. Kotikov, G. Parente, O. A. Sampayo, and J. Sanchez Guillen, Phys. Rev. Lett. 65, 1535 (1990) [Erratum-ibid. 65, 2921 (1990)]; J. Sanchez Guillen, J. Miramontes, M. Miramontes, G. Parente, and O. A. Sampayo, Nucl. Phys. B 353, 337 (1991).

[10] D. J. Gross and F. Wilczek, Phys. Rev. D 9, 980 (1974); H. Georgi and H. D. Politzer, Phys. Rev. D 9, 416 (1974); V. N. Gribov and L. N. Lipatov, Sov. J. Nucl. Phys. 15, 438 (1972) [Yad. Fiz. 15, 781 (1972)]; G. Altarelli and G. Parisi, Nucl. Phys. B 126, 298 (1977); Y. L. Dokshitzer, Sov. Phys. JETP 46, 641 (1977) [Zh. Eksp. Teor. Fiz. 73, 1216 (1977)]; K. J. Kim and K. Schilcher, Phys. Rev. D 17, 2800 (1978).

[11] E. G. Floratos, D. A. Ross, and C. T. Sachrajda, Nucl. Phys. B 129, 66 (1977) [Erratum-ibid. B 139, 545 (1978)]; G. Curci, W. Furmanski, and R. Petronzio, Nucl. Phys. B 175, 27 (1980); W. Furmanski and R. Petronzio, Phys. Lett. B 97, 437 (1980); A. Gonzalez-Arroyo, C. Lopez, and F. J. Yndurain, Nucl. Phys. B 153, 161 (1979); A. Gonzalez-Arroyo and C. Lopez, Nucl. Phys. B 166, 429 (1980); E. G. Floratos, R. Lacaze, and C. Kounnas, Phys. Lett. B 98, 89 (1981); Phys. Lett. B 98, 285 (1981); Nucl. Phys. B 192, 417 (1981).

[12] S. Moch, J. A. M. Vermaseren, and A. Vogt, Nucl. Phys. B 688, 101 (2004); Nucl. Phys. B 691, 129 (2004).

[13] A. Vogt, Comput. Phys. Commun. 170, 65 (2005).

[14] S. Moch, J. A. M. Vermaseren and A. Vogt, Phys. Lett. B 606, 123 (2005) [hep-ph/0411112]. 